THERAPEUTICS

\section{p53 restoration}

More than $50 \%$ of human tumours carry mutations in TP53, so strategies to restore the activity of this tumour suppressor in cancer cells are being actively investigated by drug companies. A team of researchers in Sweden and Russia now report the discovery of a compound that reactivates p 53 function and induces apoptosis in human cancer cells.

In the March issue of Nature Medicine, Bykov et al. describe a chemical library screen for compounds that selectively inhibit the growth of mutant TP53-expressing tumour cells. This led to the discovery of a small molecule that they called PRIMA-1 (p53 reactivation and induction of massive apoptosis), which inhibits the growth of cells expressing a number of different TP53 mutations. p53 is a transcription factor that suppresses tumour growth by several mechanisms, including induction of apoptosis. Bykov et al. show that treatment of tumour cells with PRIMA-1 caused a substantial increase in the number of cells undergoing caspase-mediated apoptosis. Furthermore, growth of tumours that carry TP53 mutations was suppressed in mouse xenografts following both intratumoral and intravenous administration of PRIMA-1.

PRIMA-1 induced apoptosis and slowed tumour growth in a mutant p53-dependent manner. TP53 mutants that are present in a number of different human cancers fail to bind the consensus DNA-binding

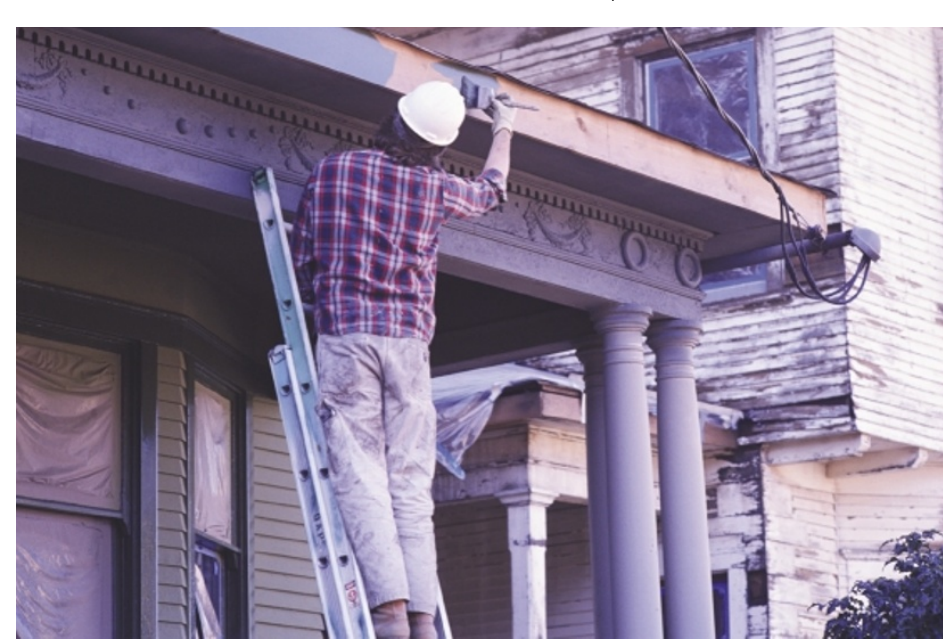

site, and Bykov et al. showed that treatment of cells with the compound restored DNA binding to 13 out of 14 TP53 mutants. PRIMA-1 also stimulated transcription of two classic p53 target genes - CDKN1A and MDM2 - only in cells that express mutant forms of TP53.

Bykov et al. are not sure about the exact molecular mechanism of PRIMA-1. Studies with conformation-specific monoclonal antibodies revealed that the drug restores the wild-type structure to mutant p53. PRIMA- 1 also rescued this conformation in the absence of other cellular proteins, indicating its ability to directly interact with p53. The authors are planning nuclear magnetic resonance and X-ray crystallography studies to further address questions about how the compound works. Other peptides and small molecules have been previously reported to protect p53 against denaturation or restore DNA binding activity to a mutant $\mathrm{p} 53$ domain. PRIMA-1 is the first molecule shown to convert p53 back into a properly folded, biologically active form.

Kristine Novak

(2) References and links ORIGINAL RESEARCH PAPER Bykov, V. J. et al. Restoration of the tumor suppressor function to mutant $\mathrm{p} 53$ by a low-molecular-weight compound. Nature Med. 8, 282-288 (2002) FURTHER READING Bullock, A. N. \& Fersht, A. R. Rescuing the function of mutant p53. Nature Rev.

WEB SITE

Department of Biosciences at the Karolinska Institute: http://www.biomed.ki.se/int//index.html Cancer 1, 68-76 (2001)

\title{
TRIAL WATCH
}

\section{Catching colon cancer}

A new method of identifying DNA mutations in faecal samples allows colorectal cancer to be non-invasively detected at very early stages. In the 31 January issue of the New England Journal of Medicine, Giovanni Traverso et al. report the development of a new assay to screen faecal samples for mutations in the adenomatous polyposis coli (APC) gene. Mutations in APC are the earliest detectable molecular abnormalities in both familial and sporadic colorectal cancer. As colon cancer cells are shed into the faeces, the authors devised a technique to detect cancer-associated mutations in stool samples.

Most cancer-associated APC mutations are clustered in a small region and interrupt transcription of the gene, leading to production of a truncated protein. The authors used an in vitro transcription-translation reaction to identify truncated APC protein within sloughed cells in stool samples. Using this assay, they were able to identify $57 \%$ of the patients with colorectal cancer or colonic polyps, and the test did not yield any false-positive results. The assay is also technically impressive, as mutant $A P C$ genes are hard to detect, comprising only $0.4-14.1 \%$ of all $A P C$ genes in the sample. Further studies are required to determine whether or not these results can be repeated on a large-scale basis, but this is an important step towards a reliable, non-invasive molecular test for colorectal cancer.

ORIGINAL RESEARCH PAPER Traverso, G. et al. Detection of $A P C$ mutations in fecal DNA from patients with colorectal tumors. N. Engl. J. Med. 346, 311-320 (2002)

\section{A promising drug for mesothelioma}

Results of a Phase II multicentre trial indicate that the ribonuclease-based drug ranpirnase (Onconase) shows promise as a treatment for patients with inoperable malignant mesothelioma. This asbestos-related cancer of the inner lining of the chest and abdomen will kill over 250,000 people in Europe alone in the next 35 years. As reported in the Journal of Clinical Oncology, the tumours either shrank or stopped growing in 41 of the 81 patients that were assessable for tumour response. The median survival time for these patients was 18.5 months - a dramatic improvement over the 6-8month life expectancy for the average mesothelioma patient. The results were especially encouraging in light of the fact that over one-third of these patients did not respond successfully to prior systemic therapy.

Ranpirnase - a ribonuclease that was developed from the eggs of a frog, Rana pipiens - interrupts protein synthesis, resulting in the inhibition of cell growth and the induction of apoptosis in cancer cells. The drug has been shown to be well tolerated in most patients, and has not been associated with the toxicity that is typically associated with chemotherapy. A randomized, controlled Phase III trial of the combination of ranpirnase with doxorubicin in patients with inoperable malignant mesothelioma, compared with doxorubicin therapy alone, is also underway in the United States and Europe.

ORIGINAL RESEARCH PAPER Mikulski, S. M. et al. Phase II trial of a single weekly intravenous dose of ranpirnase in patients with unresectable malignant mesothelioma. J. Clin. Oncol. 20, 274-281 (2002) 